\title{
Pathological approach to idiopathic interstitial pneumonias: useful points for clinicians
}

\section{Educational aims}

$>$ 1. To highlight the pathological features of the IIPS.

$>2$. To show the correlation of histology and radiology in the IIPS.

$>3$. To improve understanding of when to get a biopsy and what type of biopsy to get in the setting of an IIP.

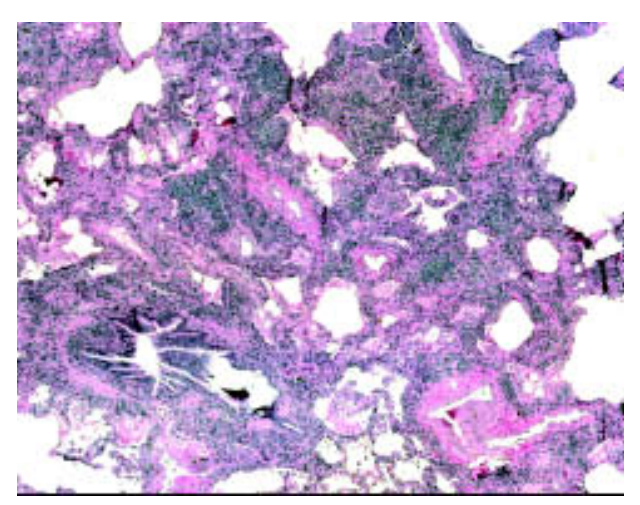

\section{Summary}

Most of the IIPs are diagnosed histologically on the basis of patterns of inflammation and fibrosis. Most require open lung biopsy for definitive histological diagnosis. There is good histological/radiological (HRCT) correlation among the IIPs, but both have their limitations. Radiology gives the overview of gross pathology, but the resolution is limited, whereas biopsy shows the histological

features but is limited by sampling; the two modalities are complementary.

Histology is not always the gold standard. Histology is a relative gold standard that is modified by other findings (particularly those of HRCT). The new ATS/ERS classification of IIPs is based on clinical-radiological-pathological correlations. Coordinating clinicalradiological-pathological data in the diagnosis of IIP is significantly better than the sum of the individual data. NSIP is still evolving.

\section{Table 1. - ATS/ERS classification of IIPS}

\begin{tabular}{|ll}
\hline Histological patterns & Clinical-radiological-pathological diagnoses \\
Usual interstitial pneumonia (UIP) & $\begin{array}{l}\text { Idiopathic pulmonary fibrosis (IPF) } \\
\text { Cryptogenic fibrosing alveolitis (CFA) }\end{array}$ \\
Nonspecific interstitial pneumonia (NSIP) & Nonspecific interstitial pneumonia (provisional) (NSIP) \\
Organising pneumonia (OP)\# & Cryptogenic organising pneumonia (COP)" \\
Diffuse alveolar damage (DAD) & Acute interstitial pneumonia (AIP) \\
Respiratory bronchiolitis (RB) & Respiratory bronchiolitis interstitial lung disease (RBILD) \\
Desquamative interstitial pneumonia (DIP) & Desquamative interstitial pneumonia (DIP) \\
Lymphocytic interstitial pneumonia (LIP) & Lymphocytic interstitial pneumonia (LIP)
\end{tabular}

": also known as bronchiolitis obliterans-organising pneumonia (BOOP); ": also known as idiopathic BOOP. 
Current classification

An American Thoracic Society (ATS)/ European Respiratory Society (ERS) Consensus Panel, co-chaired by W.D. Travis and T.E. King, recently completed a consensus classification of the idiopathic interstitial pneumonias (IIPS). Their main message was that "the IIPs comprise a number of clinicopathological entities, which are sufficiently different from one another to be designated as separate disease entities". Distinction among these diseases is based on a combination of history, physical examination, chest radiology, laboratory studies and pathology. The ATS/ERS classification is shown in table 1.

The distinction between a histological pattern (which may have a variety of causes or associations) from a clinical-radiological-pathological diagnosis is emphasised in this classification. Although it seems inappropriate in a classification of IIPS, two conditions associated with smoking, respiratory bronchiolitis interstitial lung disease (RBILD) and desquamative interstitial pneumonia (DIP), are included. The degree, pattern, character of fibrosis, and the type and the location of the cellular reaction are evaluated in making the pathological distinctions among the IIPs. The most significant finding is the extent of irreversible fibrosis, which usually takes the form of honeycomb change.

\section{Key histological features of UIP}

$>$ Dense fibrosis causing remodelling of lung architecture with frequent honeycomb fibrosis.

> Fibroblastic foci typically at the edge of the dense scars (arrows).

$>$ Patchy lung involvement.

$>$ Frequent subpleural, paraseptal and/or peribronchovascular distribution.

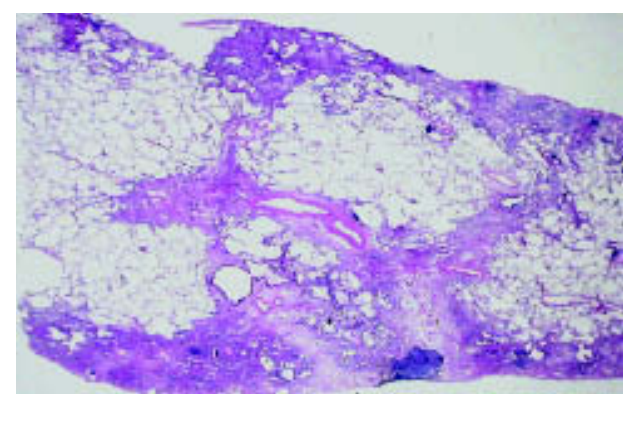

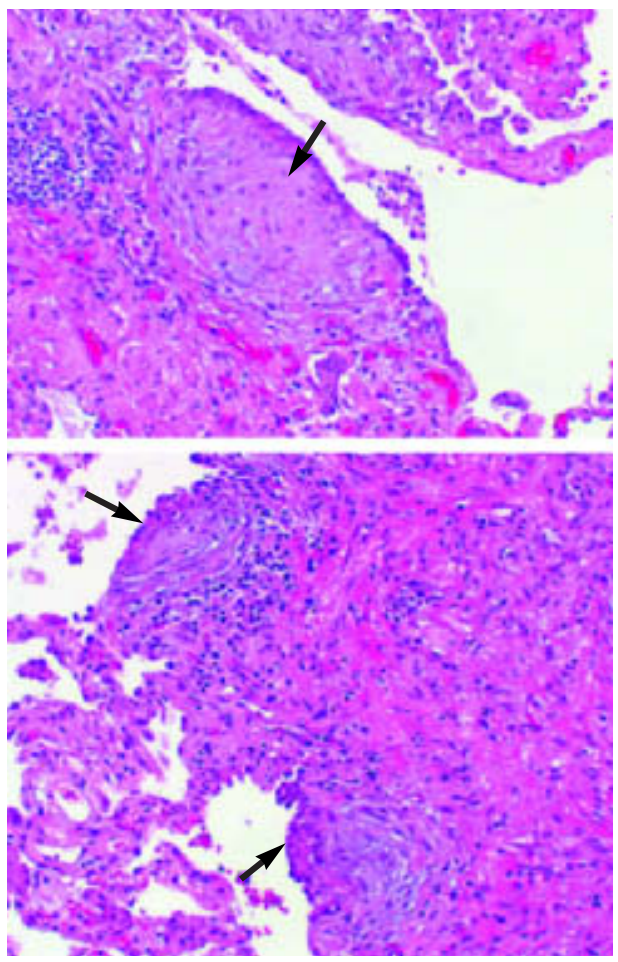

The most important lesion in the differential diagnosis of usual interstitial pneumonia (UIP) is currently non-specific interstitial pneumonia (NSIP). Retrospective studies have shown that IIPs showing an NSIP pattern have, for the most part, a better prognosis than those with UIP.

Interstitial pulmonary fibrosis (IPF) (idiopathic UIP) appears to have a stepwise progression and, in some instances, the "steps" are clinically apparent as exacerbations (of varying severity) of the underlying disease process. Acute exacerbation of IPF may manifest as a fulminant process with fever and diffuse alveolar infiltrates superimposed on background interstitial fibrosis. Histologically, these cases show features of diffuse alveolar damage or a bronchiolitis obliterans-organising pneumonia (BOOP)/organising pneumonia (OP) pattern superimposed on background chronic scarring.

\section{Key histological features of NSIP}

$>$ Cellular pattern: mild-to-moderate interstitial chronic inflammation.

$>$ Type 2 pneumocyte hyperplasia in areas of inflammation.

$>$ Fibrosing pattern: dense or loose interstitial fibrosis lacking the temporal heterogeneity pattern and/or patchy features of UIP. 
$>$ Lung architecture often appears lost by examination of haematoxylin and eosin-stained sections but relatively preserved on elasticstained sections.

$>$ Interstitial chronic inflammation: mild or moderate.

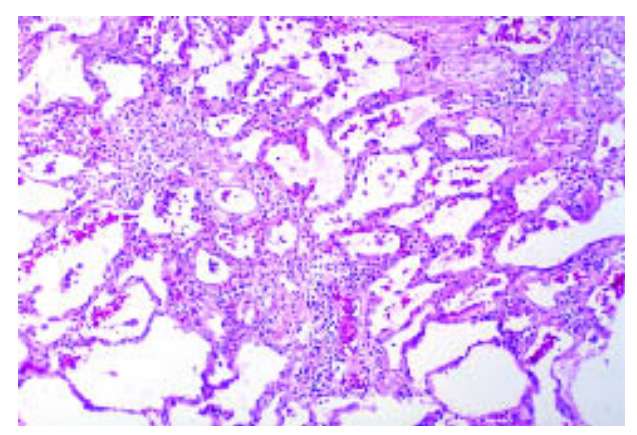

Nonspecific interstitial pneumonia (NSIP) represents a heterogenous group of interstitial pneumonias that histologically do not fit with other recognisable clinicopathological entities. It has become clear in a number of studies that, in order for designations like acute interstitial pneumonia (AIP), idiopathic $\mathrm{BOOP} /$ cryptogenic organising pneumonia (COP), UIP and DIP to be meaningful, they must be strictly defined pathologically. In keeping the criteria strict, there remain cases that do not fit easily into any of the categories. As a whole, these patients tend to fare better than patients with UIP (IPF) and AIP, and thus pathologists should not try to force individual cases into either of these categories unless they fulfil the histological features as outlined above.

Because the features are "nonspecific", the histology of NSIP is hard to define. In the original description, three categories were defined: predominantly cellular interstitial infiltrates, cellular interstitial infiltrates with some interstitial fibrosis and predominantly fibrotic. The amount of fibrosis in NSIP tends not to be great and, when extensive scarring is present, a diagnosis of UIP is most likely. In most published series, cases of interstitial pneumonia composed predominantly of interstitial infiltrates (cellular interstitial pneumonias) have not been difficult to distinguish from UIP, whereas the latter two categories have frequently been confused with UIP.

Interestingly, 5-10\% of "idiopathic" interstitial pneumonia cases called NSIP ultimately may be found to be associated with a collagen vascular disease, particularly dermatomyositis/polymyositis. NSIP is also a more common pattern among collagen vascular diseases than UIP.

\section{Key histological features of OP}

$>$ Organising pneumonia: intraluminal organisation in distal airspaces (arrows).

$>$ Patchy distribution.

$>$ Preservation of background lung architecture.

$>$ Uniform temporal appearance.

$>$ Mild interstitial chronic inflammation.

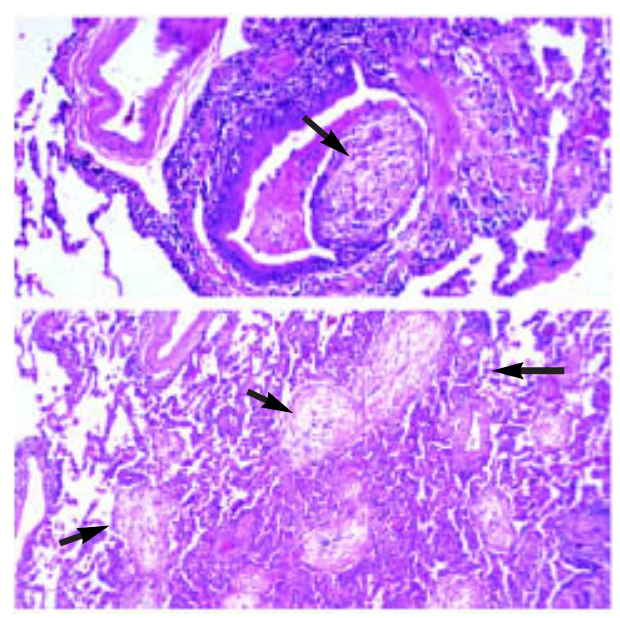

Cryptogenic organising pneumonitis (COP) represents an idiopathic interstitial pneumonia showing an organising pneumonia (OP) pattern for which no cause can be found. An OP pattern shows oedematous tufts of granulation-type tissue predominantly within airspaces, usually alveolar ducts, but often bronchioles (hence the "bronchiolitis obliterans" component of the diagnosis) as well as alveoli. There is a mild-to-moderate interstitial infiltrate in the regions of organisation usually associated with a modest type II cell proliferation, although markedly prominent type II cells are unusual. Alveolar spaces may contain foamy macrophages. Eosinophils and neutrophils are few in number. If the eosinophils are prominent, the possibility of chronic eosinophilic pneumonia or a COP/chronic eosinophilic pneumonia hybrid should be considered. There are a number of causes of an organising pneumonia pattern.

\section{Key points}

IPF has been narrowed down to a more homogeneous group of patients with a very poor prognosis (median survival $<3$ yrs) and (by definition) the histological pattern of UIP.

Histological evaluation of IIPs in general (and cases formerly interpreted clinically as IPF in particular) has resulted in subclassification into prognostically significant histopathological groupings.

Histopathological classification of interstitial pneumonias generally requires a surgical lung biopsy (exceptions: some cases of COP and AIP); clinical diagnosis may not always require a biopsy (see below).

Histological classification of interstitial pneumonias requires (by definition) histopathology. HRCT is a surrogate for surgical lung biopsy in some settings, especially UIP. The other histological patterns have less distinctive HRCT features.

In all the categories, there remain cases that are hard to classify. 
Based on the recently published ATS/ERS International Multidisciplinary Consensus Classification of Idiopathic Interstitial Pneumonias. Travis WD, King TE (CoChairs) and ATS/ERS Panel. Am J Respir Crit Care Med 2002; 165: 277-304.

\section{Key histological features of DAD}

$>$ Diffuse distribution.

$>$ Uniform temporal appearance.

$>$ Alveolar septal thickening due to organisation, usually diffuse.

> Airspace organisation (patchy or diffuse).

$>$ Hyaline membranes (focal or diffuse).

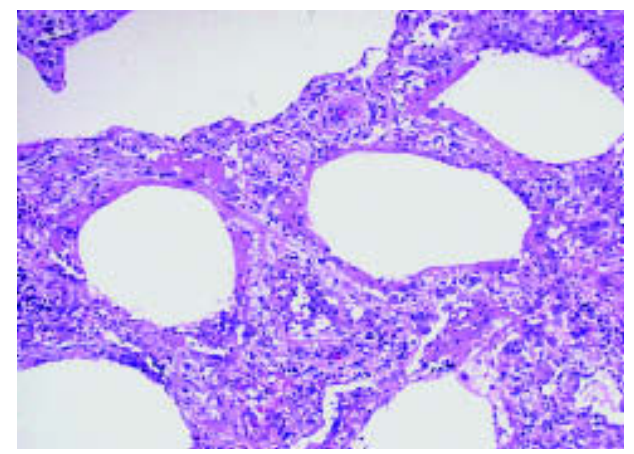

Acute interstitial pneumonia (AIP) is sometimes considered synonomous with Hamman-Rich syndrome, as originally described by Hamman and Rich, although AIP is a very rapidly evolving lesion (over days or a few weeks) whereas the original cases of Hamman and Rich had a course spanning 1-3 months and occurred prior to ventilator therapy. AIP has the appearance of acute and/or organising diffuse alveolar damage (DAD). As such, AIP is a diagnosis of exclusion of the various causes of DAD.

\section{Key histological features of RB}

$>$ Bronchiolocentric alveolar macrophage accumulation.

$>$ Mild bronchiolar and peribronchiolar fibrosis and chronic inflammation.

$>$ Macrophages with dusty brown appearance (may be positive on iron stains).

$>\mathrm{RB}$ is usually an asymptomatic microscopic finding in cigarette smokers.

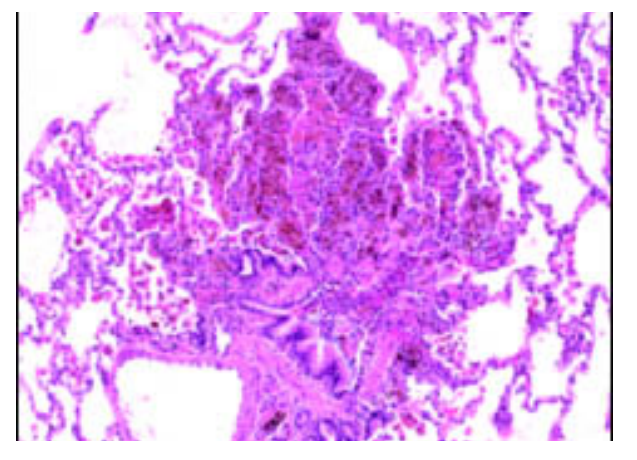

Respiratory bronchiolitis interstitial lung disease (RBILD) represents an exaggerated respiratory bronchiolitis (RB) reaction to smoking with accumulations of macrophages around respiratory bronchioles and mild associated inflammatory fibrotic and metaplastic change. The histological changes are sufficient to produce signs, symptoms and radiological findings of interstitial lung disease, and as such RBILD overlaps with DIP. This is not surprising since $~ 90 \%$ of patients in the original series of DIP smoked, and cases of RBILD and the majority of cases of DIP can now be recognised as forms of smoking-related interstitial lung disease.

\section{Key histological features of DIP}

$>$ Uniform involvement of lung parenchyma.

$>$ Prominent accumulation of alveolar macrophages (may resemble those of RB).

$>$ Mild-to-moderate fibrotic thickening of alveolar septa.

$>$ Mild interstitial chronic inflammation (lymphoid aggregates).

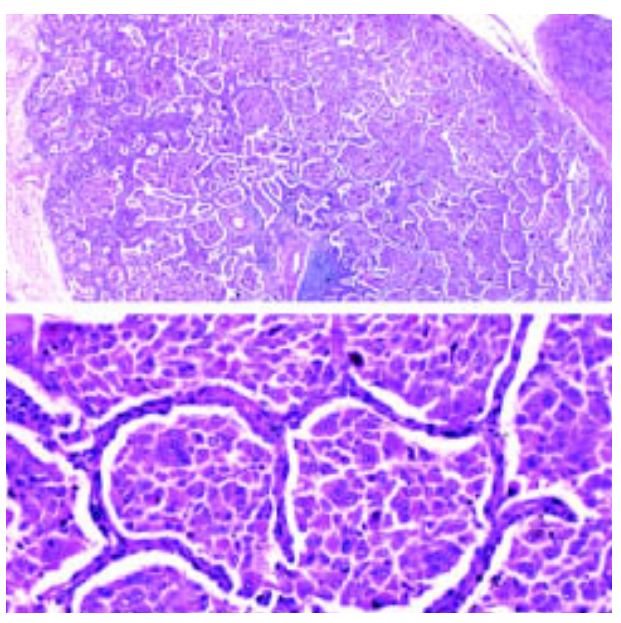

Desquamative interstitial pneumonia (DIP) is characterised by diffuse fairly uniform involvement of the lung parenchyma. There is mild-to-moderate alveolar septal widening by fibrous tissue, with a modest infiltrate that usually includes lymphocytes, plasma cells and occasional eosinophils. The most prominent feature is large accumulation of alveolar macrophages. The underlying architecture of the lung often suggests emphysema because many of the airspaces are enlarged. Honeycombing may be present, but the patchy 
irregular fibrosis with zones of honeycombing and zones of normal lung as seen in UIP is lacking.

\section{Key histological features LIP}

$>$ Diffuse interstitial infiltration.

$>$ Predominantly alveolar septal distribution.

$>$ Infiltrates comprise mostly T-cells, plasma cells and macrophages.

> Lymphoid hyperplasia (bronchoalveolar lavage hyperplasia) frequent.

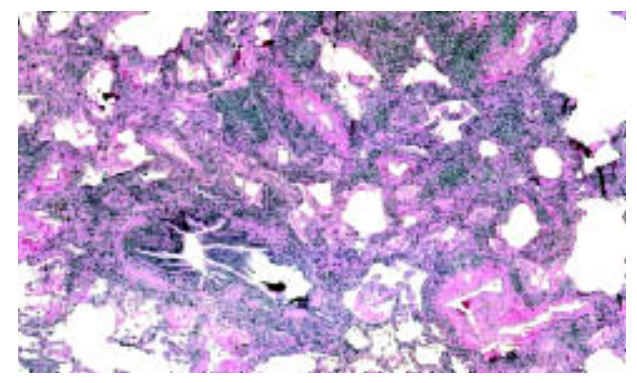

Currently lymphocytic interstitial pneumonia (LIP) includes two broad groups of lesions: 1) dense diffuse polyclonal infiltrations of lymphoid cells and 2) diffuse lymphoid hyperplasia (DLH) along lymphatic routes. There is overlap between these two basic patterns. LIP should be considered a reaction pattern with a number of causes. From a practical point of view, autoimmune diseases (particularly collagen vascular diseases) and immunodeficiency syndromes are the main lesions to be excluded when an LIP pattern or DLH are encountered.

\section{Current issues}

\section{Histological diagnosis of IIP}

Surgical lung biopsy is recommended in IIP. Exceptions include AIP and COP in which transbronchial biopsy findings consistent with DAD or OP, respectively, may suffice if clinical and radiological findings are typical.

Is biopsy always necessary? No. In clinical practice, the majority of patients with interstitial lung disease do not have biopsies (for a variety of reasons). In the case of UIP, classic HRCT findings may allow for a presumptive diagnosis of IPF in the absence of surgical lung biopsy.

Is biopsy the only gold standard? No. HRCT may provide considerably more information and trump a poor or nonrepresentative biopsy in an individual case. The importance of pathological-radiological correlation is emphasised. As a rule, while biopsy may be used to confirm the presence of significant fibrosis, HRCT better addresses the extent of fibrosis (i.e. the gross pathology).

Ground-glass change on HRCT is not always due to cellular infiltrates (airspace, interstitial or both); it may be due to fine fibrosis without coarse honeycomb change, in which case biopsy confirmation of irreversible scarring trumps the radiological impression.

As a corollary, radiological honeycombing includes only a subset of cases that show histological honeycombing. Not all histological honeycombing is seen radiologically.

\section{The problem of NSIP}

NSIP was considered a provisional entity in the ATS/ERS Consensus Classification, since it has not been adequately defined clinically, radiologically or histologically.

Initially NSIP was considered a "wastebasket diagnosis", including cases that did not fulfil the criteria for UIP (or the other IIPS). Thus, NSIP was defined in a negative way. Recently there has been an attempt (another ATS working group) to define NSIP prospectively on the basis of positive criteria. Not surprisingly, cases selected in this manner include only a proportion (less than a third) of cases originally defined as NSIP on the basis of "not UIP". Nevertheless the
Discover the original lecture given by T.V. Colby during the ERS School Course on Lung Pathology. Pathology slides available at www.breathe-cme.org 
group concluded that NSIP was an entity that required clinical, radiological and pathological input for definition and diagnosis. The issue of whether NSIP should be divided into subgroups (such as cellular, cellular/fibrosing, fibrosing) has not been resolved.

The differences in survival noted in the several series of NSIP remain to be explained. The differences are probably due to differences in the populations studied and the criteria used for the histological diagnosis on NSIP.

\section{Discordant UIP and NSIP "in" UIP}

A recent study by Flaherty et al. showed that among patients with suspected IPF who had multiple lobes biopsied, histological discordance between lobes was frequent $32 \%$ of cases); some lobes showed NSIP, whereas others showed classic UIP. The majority showed either NSIP in all lobes (27\%) or UIP in all lobes (41\%). The discordant UIP cases were classified as UIP (based on what was considered the most significant histological change), and these patients faired significantly worse than those with NSIP but better (and were somewhat younger) than those cases classified as concordant UIP. This led some to consider the possibility that (some cases of) NSIP may represent "early UIP". This problem requires further study.

The discordance between histological findings in lobes also raises a significant issue in surgical lung biopsies, namely sampling error. Even in single-lobe biopsies that show typical UIP, one may select clinical fields that qualify as NSIP, further emphasising that surgical lung biopsies should be of adequate size $(\geq 4.0 \mathrm{~cm}$ in greatest dimension would be optimal) and appropriately representative to include zones of significant fibrosis or honeycombing (to confirm the fibrotic nature of the process), in addition to less affected regions. Including only the least affected regions of the lung could lead to underdiagnosis of UIP.
The finding of NSIP-like areas in cases of UIP was recently documented by Katzenstein et al. These authors showed that 16 out of 20 explants from patients with UIP had foci resembling NSIP. The authors do not state how big the focus had to be to qualify as an "NSIP-like area", but did point out that in most of the cases $<10 \%$ of the parenchyma (which lestimate to be $<252$ $\mathrm{mm}$ on a given slide) was affected. Any casual observer knows that at medium power microscopy one can find foci resembling NSIP very commonly. Thus, this is not an issue when one defines patterns primarily at scanning power microscopy, which for most microscopes shows area $>252 \mathrm{~mm}$.

\section{New lesions on the horizon}

Yousem and Dacic recently reported a small group of chronic interstitial pneumonias under the heading of bronchiolitis with interstitial pneumonia. A somewhat similar group was presented by Churg et al. at the ATS meeting in Seattle in 2003 under the heading of intersmall airway centred fibrosis (ACF). These appear to be a group of lesions that have clinical, radiological and functional features of interstitial lung disease, yet the pathology is primarily bronchiolocentric with inflammation, fibrosis and peribronchiolar metaplasia. The cases lack histological granulomas that would suggest hypersensitivity pneumonitis. Both studies suggested that this condition could be progressive and even fatal, yet the prognosis was somewhat more favourable than for IPF. The number of cases of this condition that have been described is small and it is not clear from the two series that they include identical cases. This is an area that needs further classification. Needless to say, clinicians in Seattle were not excited about the possibility of another interstitial pneumonia! 


\section{References}

> Churg A, Jyers J, Suarez $T$, et al. Airway-centered interstitial fibrosis: a distrint form of aggressive diffuse lung disease. Am J Surg Pathol 2004; 28: 62-68.

$>$ Cordier J, Loire R, Brune J. Idiopathic bronchiolitis obliterans organizing pneumonia: definition of characteristic clinical profile in a series of 16 patients. Chest 1989; 96: 999-1004.

$>$ Cottin V, Donsbek A-V, Revel D, Loire R, Cordier J-f. Nonspecific interstitial pneumonia. Individualization of a clinicopathologic entity in a series of 12 patients. Am J Respir Crit Care Med 1998; 158: 1286-1293.

$>$ Daniil ZD, Gilchrist FC, Nicholson AG, et al. A histologic pattern of nonspecific interstitial pneumonia is associated with a better prognosis than usual interstitial pneumonia in patients with cryptogenic fibrosing alveolitis. Am J Respir Crit Care Med 1999; 160: 899-905.

> du Bois RM, Wells AU. Cryptogenic fibrosing alveolitis/idiopathic pulmonary fibrosis. Eur Respir J 2001; 18: Suppl. 32, 43S-55S.

> Hunninghake GW, Gross TJ. Idiopathic pulmonary fibrosis. N Engl J Med 2001; 345: 517-525.

> Johnston IDA, Prescott RJ, Chalmers JC, Rudd RM (for the Fibrosing Alveolitis Subcommittee of the Research Committee of the British Thoracic Society). British Thoracic Society study of cryptogenic fibrosing alveolitis: current presentation and initial management. Thorax 1997; 52: 38-44.

> Katzenstein A, Fiorelli RF. Nonspecific interstitial pneumonia/fibrosis: histologic features and clinical significance. Am J Surg Pathol 1994; 18: 136-147.

> Katzenstein A, Myers JL, Mazur M. Acute interstitial pneumonia: a clinicopathologic, ultrastructurol, and cell kinetic study. Am J Surg Pathol 1986; 10: 256-267.

> Katzenstein ALA, Myers JL. Idiopathic pulmonary fibrosis: state of the art. Am J Respir Crit Care Med 1998; 157: 1301-1315.

> Katzenstein ALA, Zisman DA, Litzky LA, Nguyen BT Kotloff RM. Usual interstitial pneumonia. Histologic study of biopsy and explont specimens. Am J Surg Pathol 2002; 26: 1567-1577.

> King TE, Costobel U, Cordier $F$, et al. Idiopathic pulmonary fibrosis: diagnosis and treatment. International Consensus Statement. Am J Respir Crit Care Med 2000; 161: 646-664.

$>$ Kondoh Y, Taniguchi H, Kawabata Y, Yokoi T, Suzuki K, Takagi K. Acute exacerbation in idiopathic pulmonary fibrosis. Chest 1993; 103: 1808-1812.

> Moon J, du Bois RM, Colby N, Hansell DM, Nicholson AG. Clinical significance of respiratory bronchiolitis on open lung biopsy and its relationship to smoking related interstitial lung disease. Thorax 1999; 54: 1009-1014.

$>$ Myers JL. NSIP, UIP, and the ABCS of idiopathic interstitial pneumonias. Eur Respir J 1998; 12: 1003-1004.

> Myers IL, Veal CF Jr, Shin MS, Katzenstein AL. Respiratory bronchiolitis causing interstitial lung disease: a clinicopathologic study of six cases. Am Rev Respir Dis 1987, 135: 880-884.

> Nagai S, Kitoichi M, Itoh H, Nishimura K, Isumi T, Colby TV. Idiopathic nonspecific interstitial pneumonia/fibrosis: comparison with idiopathic pulmonary fibrosis and BO0R. Eur Respir J 1998; 12: 1010-1019.

> Nicholson AG, Colby TV, du Bois RM, Hansell DM, Wells AU. The prognostic significance of histological pattern of usual interstitial pneumonia in patients presenting with clinical entity of cryptogenic fbrosing alveolitis. Am J Respir Crit Care Med 2000; 162: 2213-2217.

> Ryu JH, Colby TV, Hartman TE. Idiopathic pulmonary fibrosis: current concepts. Mayo Clin Proc 1998; 73: 1085-1101.

> Ryu JH, Colby TV, Hartman TE, Vassollo R. Smoking related interstitial lung diseases: a concise review. Eur Respir J 2001;17:122-132.

> Yousem SA, et al. Respiratory bronchiolitis associated interstitial lung disease and its relationship to desquomative interstitial pneumonia. Mayo Clin Proc 1989, 64: 1373-1380.

> Yousem SA, Docic S. Idiopathic bronchiolocentric interstitial pneumonia. Mod Pathol 2002; 15: 1148-1153.

\section{Recommended reading}

> Bjoroker JA, Ryu JH, Edwin MK, et al. Prognostic significance of histolopathologic subsets in idiopathic pulmonary fibrosis.

Am J Respir Crit Care Med 1998; 157: 199-203.

> Flaherty KR, Travis WD, Colby TV, et al. Histological variability in patients with suspected IPF. Clinical implications. Eur Respir J 2004 (in press).

> Hunninghake $G$, Zimmerman $M B$ Schwartz $D$, et al.

Utility of lung biopsy for the diagnosis of idiopathic pulmonary fibrosis.

Am J Respir Crit Care Med 2001; 164: 193-196.

$>$ Travis WD, King TE (Co-Chairs) and ATS/ERS Panel.

ATS/ERS International Multidisciplinary Consensus Classification of Idiopathic Interstitial Pneumonias.

Am J Respir Crit Care Med 2002; 165 : 277-304. 